Original Article

\title{
MODULATORY EFFECTS OF BONE MARROW-DERIVED MESENCHMAL STEM CELLS ON CYCLOPHOSPHAMIDE INDUCED HEPATOTOXICITY IN CARCINOMA MICE
}

\author{
ASMAA MAGDY ZAAZAA ${ }^{*}$, BOSY AZMY ABD EL-MOTELP1
}

${ }^{1}$ Assistant Professor of Physiology at Department of Zoology, Faculty of Women for Arts, Science and Education, Ain Shams University, Asmaa Fahmy Street Heliopolis, Cairo, Egypt

Email: asmaa.zaazaa@women.asu.edu.eg

Received: 23 Jan 2020, Revised and Accepted: 04 Mar 2020

\begin{abstract}
Objective: Cyclophosphamide (CPA) is a chemotherapeutic agent, induces hepatotoxicity as one of its side effects. Therefore, the present work was designed to investigate the protective role of bone marrow-derived mesenchymal stem cells (BM-MSCs) on CPA-induced hepatotoxicity in Ehrlich Ascites Carcinoma bearing-mice (EAC) and to test whether BM-MSCs influences the antitumor properties of the CPA.

Methods: The hepatoprotective effects of BM-MSCs (single dose of $100 \mu \mathrm{l}$ of a cell suspension containing allogenic BM-MSCs, i. v.) was evaluated in a model of hepatotoxicity by CPA (10 mg/kg/d i. p.) in EAC-female mice for one month. The anti-tumor activities of CPA and BM-MSCs were assessed by measuring mean tumor weight, mean survival time and the increase in life span. Moreover, ALT, AST, GGT, MDA, GSH, SOD, IL-6, IL10, caspase-3 and $\mathrm{Bcl} 2$ were measured.

Results: The i. p. administration of CPA and BM-MSCs resulted in significant reductions in tumor size and mean tumor weight as well as caused concurrent significant increases in the life span as compared to the EAC mice. Furthermore, BM-MSCs ameliorated the liver enzyme markers namely ALT, AST, GGT, and hepatic oxidative stress through inhibition of MDA level that correlated with significant improvement in antioxidant status via increasing GSH and SOD levels as compared to both EAC and EAC+CPA groups. Moreover, BM-MSCs treatment significantly reduced the inflammatory marker level IL-6 as well as increment the level of IL-10 with subsequent decreases apoptosis via a depletion in the caspase-3 associated with an enhancement in the level of Bcl2 as compared to EAC group and EAC+CPA group. Minor histological lesions were observed in the liver tissue sections of mice treated with CPA and BM-MSCs as compared to the high histological lesions observed in the liver of the EAC group and CPA treated group.
\end{abstract}

Conclusion: These results concluded that the combination treatment of BM-MSCs with CPA exhibited promising potential antitumor efficacy with greater safety than CPA treatment alone in mice via its antioxidant, anti-inflammatory and antiapoptotic effects.

Keywords: Hepatotoxicity, Ehrlich, Oxidative stress, Inflammation, Apoptosis

(C) 2020 The Authors. Published by Innovare Academic Sciences Pvt Ltd. This is an open access article under the CC BY license (http://creativecommons.org/licenses/by/4.0/) DOI: http://dx.doi.org/10.22159/ijpps.2020v12i4.36916. Journal homepage: https://innovareacademics.in/journals/index.php/ijpps

\section{INTRODUCTION}

Cancer is one of the greatest serious diseases everywhere throughout the world. Cancer therapy and its managing depend fundamentally on the utilization of chemotherapeutic agents. These agents mainly target the rapidly proliferating neoplastic cells in cancer patients. But chemotherapy is always associated with a severe form of toxic manifestations [1]. Solid and the ascetic types of Ehrlich ascites carcinoma (EAC) are undifferentiated tumors usually used to assess different lines of cancer therapeutics [2].

Cyclophosphamide (CPA) is used as a chemotherapeutic drug [3] Numerous antineoplastic conventions require high doses of CPA that are correlated with acute side effects, such as hepatotoxicity, cardiotoxicity, nephrotoxicity, and immunotoxicity [4]. Preceding studies have exhibited that CPA could produce substantial reactive oxygen species (ROS) and add to succeeding raised concentrations of highly cytotoxic oxidative stress-resulting lipid peroxidation aldehydes, such as acrolein and malondialdehyde (MDA) [5]. CPA was demonstrated that the imponderables among oxidant and antioxidant condition lead to the creation of pro-inflammatory mediators which participate in liver damage after CPA injection [6]. Hepatotoxicity has been recorded in patients injected intravenously with a low dose of CPA [7]. Preceding studies have indicated that liver toxicity is a significant issue related to cyclophosphamide application [8]

Antioxidant agents are believed useful to alleviate oxidative stress. In like manner, a blend of a treatment program with potent and safe antioxidants could be an advantageous approach to lessen CPA-induced toxicity [9]. Mesenchymal stem cells (MSCs) are multipotent stem cells that were initially isolated from the bone marrow (BM-MSCs). BM-MSCs can differentiate into cells of the 3 germ layers, including endothelial cells of blood vessels, smooth muscle cells, adipocytes, nerve cells, chondrocytes, cardiomyocytes, and hepatocytes. Furthermore, they can reduce the immune response brought about by $\mathrm{T}$ lymphocytes in allogeneic transplantation [10]. The therapeutic effects of transplanted MSCs have been associated with the following mechanisms: (1) differentiation to parenchymal cell; (2) production of trophic features that encourage proliferation and differentiation of local progenitor cells; (3) neovascularization; and (4) immunomodulation [11]

Therefore, the present work was designed to investigate the protective role of bone marrow-derived mesenchymal stem cells (BM-MSCs) on CPA induced hepatotoxicity in EAC mice and to test whether BM-MSCs influences the antitumor properties of the CPA.

\section{MATERIALS AND METHODES}

Chemicals

CPA was supplied from Sigma-Aldrich Chemicals Co., (USA) and was prepared in sterile physiological saline (SPS). All the other chemicals were of high purity grade in agreement with international standards.

\section{Animals}

Because Ehrlich Ascites Carcinoma cells were stated to demonstrate greater initial growth and total cell count in female mice than male mice [12]. Thus, the current study carries out on female mice that were acquired from the National Cancer Institute, Cairo University.

Animals Six to eight-week-old female BALB/c mice with an average body weight of $25-30 \mathrm{~g}$ were used and were supplied from the Animal House Colony of the National Research Centre, Giza, Egypt. 
The mice were left for one week before to the experiments initiated to adapt to the animal house environments under normal dark/light cycle and were provided diets and water ad libitum. The experimental protocols were carried out corresponding to the guidelines for the animal experiment, which was approved by the Ethical Committee of Medical Research of National Research Centre, Giza, Egypt (registration number: 13/165).

\section{Tumor cell line}

The Ehrlich Ascites Carcinoma (EAC) cells were obtained from the National Cancer Institute (NCI) (Cairo, Egypt). The initial inoculation of EAC cells was kindly supplied by the National Cancer Institute (Cairo University, Egypt). EAC cells were thereafter propagating in our laboratory by weekly intraperitoneal (IP) injection of $2.5 \times 10^{6}$ cells per mouse, which was carried out according to the method recommended by the Egyptian National Cancer Institute, Cairo University. Cells were counted before injection using the bright-line hemocytometer and dilutions were made by physiological saline, and the desired number of cells was injected in a volume of $0.5 \mathrm{ml}$ per mouse.

\section{Cell viability}

On the 7th day of the initial inoculation of EAC, ascitic fluid was collected and subjected to a trypan blue dye exclusion method for estimation of cell viability [13]. The number of viable cells (unstained) was counted under a microscope and average from 5 squares was taken. The cells which took trypan blue were deemed non-viable (fig. 1). The concentration of viable cells (expressed as follows: $\mathrm{C}=\mathrm{T} \times \mathrm{D} \times 10^{4}$ ), where $\mathrm{C}=$ concentration of viable cells $/ \mathrm{ml}$, and $\mathrm{T}=$ Average no. of viable cells per square, and $\mathrm{D}=$ dilution factor.
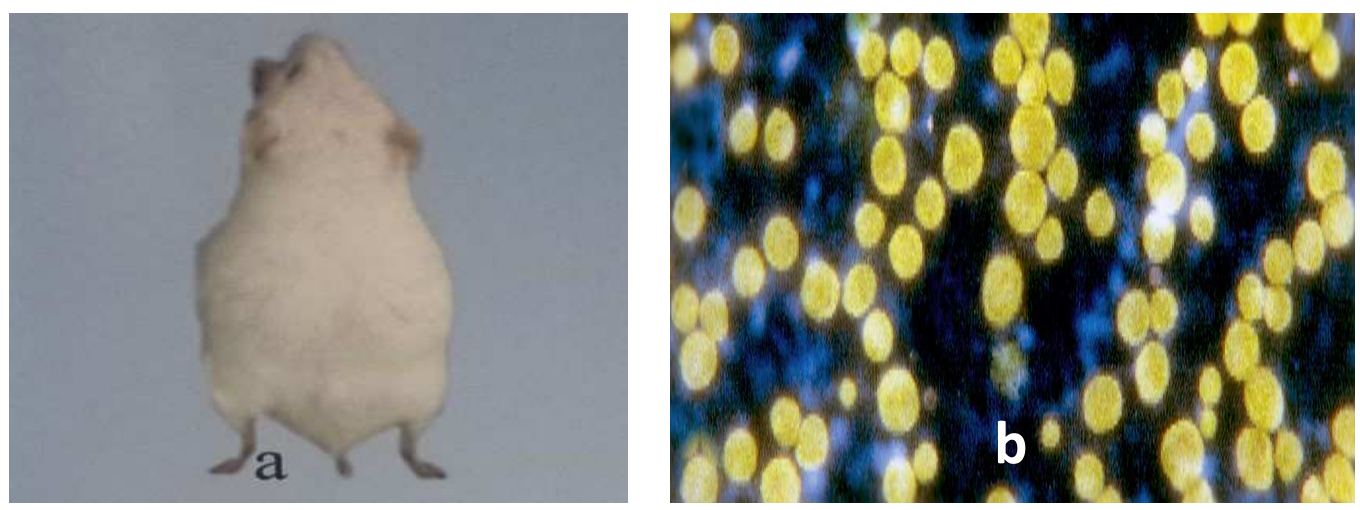

Fig. 1: (a) Showing the culture of EAC in vivo (in abdominal cavity of female mouse). (b) Showing the normal EAC cells X200

\section{Preparation and isolation of BM-derived MSCs}

Bone marrow was collected by flushing the tibiae and femurs of $6 \mathrm{w}$ old male Wistar rats with phosphate-buffered saline (PBS). Bone marrow cells were plated in Dulbecco's modified Eagle's medium (DMEM) supplemented with $10 \%$ fetal bovine serum (FBS). Nucleated cells were isolated with a density gradient and resuspended in complete culture medium supplemented with $1 \%$ penicillin-streptomycin. Cells were incubated at $37{ }^{\circ} \mathrm{C}$ in $5 \%$ humidified $\mathrm{CO} 2$ for $12 \mathrm{e} 14 \mathrm{~d}$ as primary culture or upon the formation of large colonies. When large colonies developed (80e90\% confluence), cultures were washed twice with phosphate buffer saline (PBS) ( $\mathrm{pH} \mathrm{7)}$ and the cells were trypsinized with $0.25 \%$ trypsin in $1 \mathrm{mmol}$ EDTA for $5 \mathrm{~min}$ at $37^{\circ} \mathrm{C}$. After centrifugation at $3000 \mathrm{rpm}$ for $10 \mathrm{~min}$, cells were re-suspended in serumsupplemented medium and incubated in $50 \mathrm{~cm} 2$ culture flasks. The resulting cultures were referred to as first-passage cultures [14]. Cells were identified as being MSCs by their morphology or fusiform shape, adherence [15] and Florescent Analysis Cell Sorting (FACS) by detection of CD29+, CD90+, and CD34-specific to MSCs.

\section{FACS analysis}

Following short centrifugation, cells were re-suspended in wash buffer (BD Biosciences, Germany). $300 \mathrm{ml}$ of cell suspension was incubated with antibodies compared to CD29, CD34 and CD90 conjugated with Allophycocyanin (APC), Cyanine 5 (CY5), Phycoerythrin (PE) and Fluorescein isothiocyanate (FITC) dyes respectively for $45 \mathrm{~min}$ at room temperature. Flow cytometry was executed on a FACS Caliber (BD Biosciences, Germany) and Cell Quest software was sourced for analysis.

\section{Tracking of stem cells}

MSCs cells were gathered through the 4th passage. Then, cells were trypsinized and were placed into a single cell suspension. $2 \times 107$ single cells were placed in a falcon tube, washed once applying culture medium free of serum then cells were centrifuged (400xg) for 5 min. Finally, cells were labeled with PKH26 fluorescent linker dye (according to the manufacturer's protocol) and examined using fluorescence microscopy (Sigma-Aldrich, Saint Louis, USA).

\section{Ehrlich solid carcinoma induction}

Ehrlich Solid Carcinoma (ESC) was induced into forty female mice using Ehrlich Ascites Carcinoma (EAC) cells obtained from the ascitic fluid of female mice bearing EAC. On day zero about $2.5 \times 10^{6}$ viable EAC cells present in $0.2 \mathrm{ml}$ of diluted ascitic fluid (1:10) with saline was injected intramuscularly in the left thigh of each mouse to induce solid tumors [16].

\section{Experimental protocol}

\section{Anti-tumor studies}

The anti-tumor activities of CPA or/and BM-MSCs were assessed on 192 mice bearing solid Ehrlich Carcinoma that were classified into two main groups, the first group 32 mice which were used to determine the mortality rate and subdivided into four groups $(n=8)$ as flow:

Gr.1: female mice inoculated with EAC cells $\left(2.5 \times 10^{6}\right.$ viable EAC $)$ intramuscularly in the left thigh of each mouse to induce solid tumors and served as (EAC group).

Gr.2: female mice inoculated with EAC cells $\left(2.5 \times 10^{6}\right.$ viable EAC), Ten days after inoculating the EAC cells (when the tumor becomes papale), the animals treated with CPA $(10 \mathrm{mg} / \mathrm{kg} / \mathrm{d}$ i. p.) for one month [17] and served as (EAC+CPA) group.

Gr.3: female mice inoculated with EAC cells $(2.5 \times 106$ viable EAC), Ten days after inoculating the EAC cells (when the tumor becomes papale), the animals injected with BM-MSCs i. v. by a single dose of $100 \mu \mathrm{l}$ of a cell suspension containing allogenic BM-MSCs from rats at the moment of the boost (when BM-MSCs were collected) [18] then lifted untreated for one month and served as (EAC+BM-MSCs) group.

Gr4: female mice inoculated with EAC cells $\left(2.5 \times 10^{6}\right.$ viable EAC), Ten days after inoculating the EAC cells (when the tumor becomes papale), the animals treated with both CPA and BM-MSCs at the same previous doses and served as (EAC+CPA+BM-MSCs) group.

The second group 160 mice were used to calculate the mean tumor weight (MTW) until the end of the study and were classified into four equal-sized groups $(n=40)$ as flow: 
Gr1: EAC group, Gr2: EAC+CPA group, Gr3: EAC+BM-MSCs and Gr4: EAC+CPA+BM-MSCs (the animals in these groups were injected as mentioned before). Then, these groups were left untreated until the end of the experiment. The weight of the solid tumor was determined by killing three animals every week and by taking the mean tumor weight of control (MTWc) and mean tumor weight of test (MTWt). The cumulative mean survival time of control (MSTc), the cumulative mean survival time of test (MSTt) the increase in life span (ILS, \%) and the tumor growth inhibition ratio (T/C \%) were recorded as given by Fahim et al. [19], where: MST is the days in which animals were lived only.

$$
\begin{aligned}
\text { ILS } \% & =\frac{\text { MSTt }}{\text { MSTC }} \times 100 \\
(T / C \%) & =\frac{\text { MTWc-MTWt }}{\text { MTWc }} \times 100
\end{aligned}
$$

\section{Biochemical and histopathological studies}

40 animals were assigned into five groups, each containing 8 mice as follow: Gr1: mice received i. p. injection of physiological saline and served as a normal control group (Con), Gr2: EAC group, Gr3: EAC+CPA, Gr4: EAC+BM-MSCs and Gr5: EAC+CPA+BM-MSCs (the animals in these groups from Gr2-Gr5 were injected as mentioned before).

\section{Samples collection}

At the end of the experiment, the mice were fasted overnight, subjected to anesthesia by diethyl ether and sacrificed by cervical dislocation, and a midline abdominal incision was performed, and whole liver of each animal was rapidly dissected out, thoroughly washed with ice-cold isotonic saline, blotted dry and then weighed. Each liver was divided into two portions. One portion was immediately homogenized to give $10 \%(\mathrm{w} / \mathrm{v})$ homogenate in icecold medium containing phosphate buffer (pH: 7.4). The homogenate was centrifuged at $1800 \times \mathrm{g}$ for $10 \mathrm{~min}$ at $4{ }^{\circ} \mathrm{C}$. The supernatant $(10 \%)$ was separated and stored at $-20{ }^{\circ} \mathrm{C}$ for the biochemical determinations. The second portion of the liver was fixed in $10 \%$ formalin saline for histopathological investigation.

\section{Biochemical determinations}

Hepatic total protein level was determined by the colorimetric method of Lowry et al. [20]. Hepatic alanine aminotransferase (ALT) and aspartate aminotransferase (AST) activity were determined by the colorimetric method using the Salucea kit (The Netherlands) according to the method described by Young [21]. Hepatic gamma-glutamyl transferase (GGT) was measured by standard methodology using a commercially available kit provided by the Biodiagnostic Company (Giza, Egypt).

\section{Assessment of oxidative stress}

Oxidative stress was estimated using the following parameters: Hepatic MDA content was determined by a colorimetric method using the Biodiagnostic kit (Egypt) following the method of Satoh [22]. The assay of reduced glutathione (GSH) level in liver tissue was performed using the Biodiagnostic kit purchased from the Biodiagnostic Company (Giza, Egypt). Which is based on the spectrophotometric method of Beutler et al. [23]. Hepatic superoxide dismutase activity (SOD) was determined by a colorimetric method as described by Nishikimi et al. [24] using a diagnostic kit supplied by Biodiagnostic Company (Giza, Egypt).

\section{Determination of inflammatory and anti-inflammatory markers}

The levels of biochemical parameters in liver homogenate were measured by ELISA assay using a commercially available IL- 6 and IL10 ELISA kits provided from (eBioscience Inc., San Diego, CA, USA), following the manufacturer's instructions.

\section{Estimation of apoptotic and anti-apoptotic markers}

The activity of Caspase-3 was measured in hepatic tissue by assay (ELIZA) technique using Gscience kits purchased from Glory Science Co., Ltd, USA, according to the manufacturer's instruction. Hepatic $\mathrm{Bcl} 2$ content was estimated by using ELISA kit for quantitative detection of human Bcl-2 purchased from eBioscience Co., Vienna, Austria, Europe according to the method of Barbareschi et al. [25].

\section{Histopathological investigation}

After the fixation of liver specimens in formalin saline (10\%) for 24 $h$., washing in tap water was done, and then the liver samples were subjected to serial dilutions of alcohol (methyl, ethyl, and absolute ethyl) for dehydration. Afterward, liver specimens were cleared in xylene and embedded in paraffin wax at $56^{\circ}$ in a hot air oven for 24 h. Paraffin wax tissue blocks were submitted for sectioning at $4 \mu \mathrm{m}$ by Sledge microtome. The obtained tissue sections were collected on glass slides, deparaffinized, and stained with hematoxylin and eosin [26]. After staining, the slides were viewed with an Olympus $\mathrm{CH}$ (Japan) light microscope [27].

\section{Statistical analysis}

In the present study, all results were expressed as meanıstandard error of the mean. Statistical Package for the Social Sciences program, version 14.0 was used to compare significance between every two groups. The difference was considered as statistically significant when $\mathrm{p}<0.05$. Percentage difference representing the percent of variation for the corresponding control group was calculated according to the following formula:

$$
\% \text { Difference }=\frac{\text { Treated value-control value }}{\text { Control value }} \times 100
$$

\section{RESULTS}

Cell surface marker expression analysis, characterization using flow cytometry and tracking of stem cells

The immunophenotype of BM-MSCs cells was analyzed by flow cytometry. BM-MSCs cells were negative for the hematopoietic marker CD34 (fig. 2A), while powerfully positive for mesenchymal stem cell markers including CD29 (fig. 2B) and CD90 (fig. 2C). The blue histograms represent antibody labeled cells while the grey histogram shows the profile of the isotype control. BM-MSCs were labeled by PKH26 to track its engraftment in the liver tissue (fig. 2D and 2E).
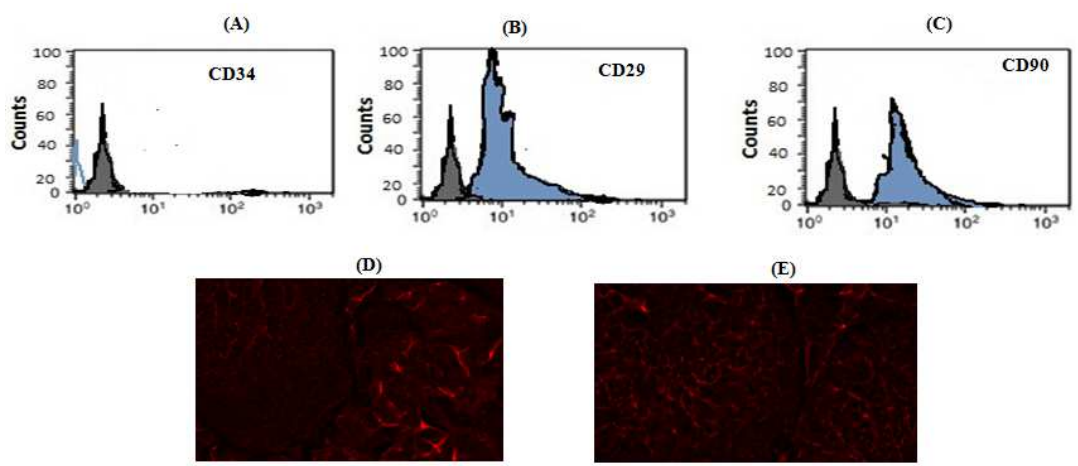

Fig. 2: Immunophenotype of BM-MSCs was examined by flow cytometry. BM-MSCs cells were negative for the hematopoietic marker CD34 (A), while strongly positive for mesenchymal stem cell-specific markers including CD29 (B) and CD90 (C). The blue histograms represent antibody labeled cells while the grey histogram shows the profile of the isotype control. EAC+BM-MSCs group showing PKH26-labelled injected stem cells engrafted in liver tissue (D) and EAC+CPA+BM-MSCs group showing PKH26-labelled injected stem cells engrafted in liver tissue (E) 


\section{The anti-tumor studies}

The treatment of EAC mice with the CPA, BM-MSCs or CPA and BM-MSCs together resulted in an increase in the life span (ILS \%), which reached to $153.8 \%, 123 \%$, and $169.2 \%$, respectively, compared to the EAC mice (table 1). No marked difference in the mean tumor weight was found in the EAC group treated with BM-MSCs (5.25g) compared to the EAC group (5.48 g). Meanwhile, Treatment involving CPA and CPA+BM-MSCs revealed highly growth retardation in the mean tumor weight $(3.41 \mathrm{~g})$ and (2.98 g), respectively (table 1and fig. 3). Moreover, the most elevation of the tumor growth inhibition ratio (T/C \%) was noted in the CPA+BM-MSCs group as compared to the EAC group (fig. 4, 5).

Table 1: The anti-tumor activities of CPA or/and BM-MSCs solid Ehrlich carcinoma mice

\begin{tabular}{|c|c|c|c|c|c|c|c|c|}
\hline \multirow{2}{*}{$\begin{array}{l}\text { PTI } \\
\text { (days) }\end{array}$} & \multicolumn{2}{|l|}{ EAC } & \multicolumn{2}{|c|}{ EAC+CPA } & \multicolumn{2}{|c|}{ EAC+BM-MSCS } & \multicolumn{2}{|c|}{ EAC+CPA+BM-MSCS } \\
\hline & $\mathbf{M}$ & MTW \pm SE & $\mathbf{M}$ & MTW \pm SE & $\mathbf{M}$ & MTW \pm SE & $\mathbf{M}$ & MTW \pm SE \\
\hline 10 & $0 / 8$ & $1.19 \pm 0.02$ & $0 / 8$ & $1.13 \pm 0.08$ & $0 / 8$ & $1.01 \pm 0.07$ & $0 / 8$ & $1.21 \pm 0.03$ \\
\hline 17 & $0 / 8$ & $2.11 \pm 0.03$ & $0 / 8$ & $1.62 \pm 0.21$ & $0 / 8$ & $1.93 \pm 0.16$ & $0 / 8$ & $1.31 \pm 0.14$ \\
\hline 24 & $0 / 8$ & $3.23 \pm 0.21$ & $0 / 8$ & $2.33 \pm 0.24$ & $0 / 8$ & $2.84 \pm 0.16$ & $0 / 8$ & $1.47 \pm 0.06$ \\
\hline 30 & $2 / 8$ & $4.55 \pm 0.33$ & $0 / 8$ & $2.58 \pm 0.21$ & $0 / 8$ & $3.71 \pm 0.03$ & $0 / 8$ & $1.72 \pm 0.19$ \\
\hline 36 & $3 / 8$ & $6.54 \pm 0.41$ & $0 / 8$ & $2.94 \pm 0.08$ & $1 / 8$ & $4.22 \pm 0.21$ & $0 / 8$ & $2.23 \pm 0.13$ \\
\hline 42 & $5 / 8$ & $7.13 \pm 0.36$ & $0 / 8$ & $3.25 \pm 0.42$ & $2 / 8$ & $6.57 \pm 0.26$ & $0 / 8$ & $2.82 \pm 0.19$ \\
\hline 48 & $6 / 8$ & $8.52 \pm 0.25$ & $0 / 8$ & $3.82 \pm 0.29$ & $3 / 8$ & $7.25 \pm 0.18$ & $0 / 8$ & $3.22 \pm 0.18$ \\
\hline 54 & $8 / 8$ & $9.82 \pm 0.45$ & $2 / 8$ & $4.23 \pm 0.32$ & $5 / 8$ & $7.91 \pm 0.20$ & $0 / 8$ & $3.68 \pm 0.28$ \\
\hline 60 & & & $3 / 8$ & $4.60 \pm 0.08$ & $6 / 8$ & $8.22 \pm 0.26$ & $1 / 8$ & $3.91 \pm 0.12$ \\
\hline 66 & & & $5 / 8$ & $5.29 \pm 0.20$ & $8 / 8$ & $8.92 \pm 0.31$ & $3 / 8$ & $4.32 \pm 0.12$ \\
\hline 72 & & & $8 / 8$ & $5.81 \pm 0.36$ & & & $6 / 8$ & $4.71 \pm 0.32$ \\
\hline 78 & & & & & & & $8 / 8$ & $5.23 \pm 0.32$ \\
\hline MTW & 5.48 & & 3.41 & & 5.25 & & 2.98 & \\
\hline MST (days) & 39 & & 60 & & 48 & & 66 & \\
\hline ILS\% & - & & 153.8 & & 123 & & 169.2 & \\
\hline $\mathrm{T} / \mathrm{C} \%$ & - & & 37.77 & & 4.05 & & 45.62 & \\
\hline
\end{tabular}

$\mathrm{PTI}=$ Post tumor inoculation. $\mathrm{M}=$ Mortality. MTW= Mean tumor weight. MST (days)= Mean survival time. $\mathrm{ILS}=\mathrm{Increase}$ of life span. $T / C=$ Tumor inhibition ratio.

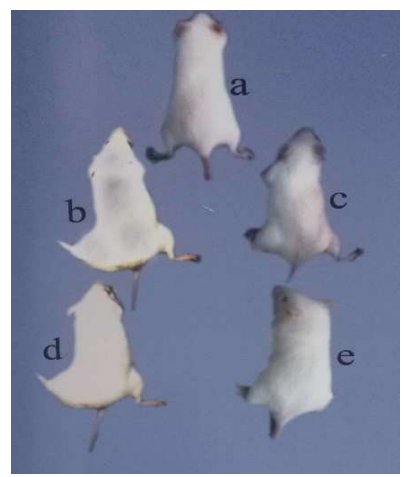

Fig. 3: Showing the anti-tumor activities of CPA or/and BM-MSCs on solid EAC compared to the control group (after forty days from inoculation of EAC). (a) normal control mouse, (b) mouse bearing tumor, (c) mouse bearing tumor and treated with CPA, (d) mouse bearing tumor and treated with BM-MSCs and (e) mouse bearing tumor and treated with CPA and BM-MSCs

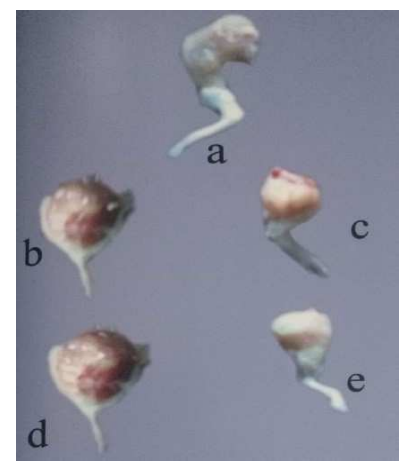

Fig. 4: Showing the morphological changes in the legs bearing tumor after treatment with CPA or/and BM-MSCs compared to control leg (after forty days from inoculation of EAC). (a) normal leg of control mouse, (b) leg of a mouse bearing tumor, (c) leg of a mouse bearing tumor and treated with CPA, (d) leg of a mouse bearing tumor and treated with BM-MSCs and (e) leg of a mouse bearing tumor and treated with CPA and BM-MSCs

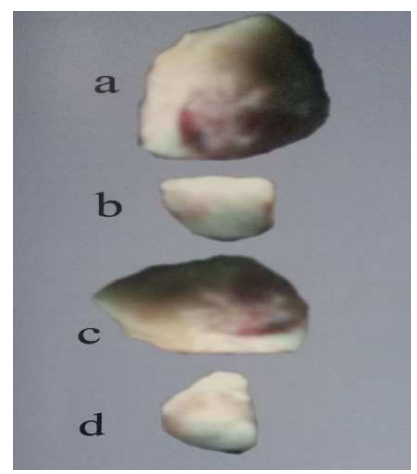

Fig. 5: Showing the reduction in the tumor size after treatment with CPA or/and BM-MSCs compared to the EAC without any treatment (after tumor isolation from the leg) after forty days from inoculation of EAC. (a) tumor of a mouse bearing EAC without treatment, (b) tumor of a mouse bearing EAC and treated with CPA, (c) tumor of a mouse bearing tumor and treated with BM-MSCs and (d) tumor of mouse bearing EAC and treated with CPA and BM-MSCs

Effects of treatment with CPA or/and BM-MSCs on Liver functions in EAC mice treated with $\mathrm{CP}$

The EAC mice (table 2) showed a generalized disturbance in all measured hepato-specific markers comparable to control $(\mathrm{P}<0.05)$. Treatment of EAC mice with CPA resulted in an increment in hepatic levels of AST, ALT, and GGT compared to tumor group. These levels of liver enzymes were declined in EAC mice treated with BM-MSCs. Otherwise, treatment of EAC mice with both CPA and BM-MSCs resulted in significant depletion $(\mathrm{P}<0.05)$ in the hepatic levels of AST, ALT, and GGT verses to both of EAC and EAC+CPA groups.

Effects of treatment with CPA or/and BM-MSCs on oxidative stress markers in EAC mice

Data in table 3 showed the effect of treatment with CPA, BM-MSCs, and their combination on MDA level and enzymatic antioxidants activities of EAC mice. The level of hepatic MDA level was significantly augmented $(\mathrm{p}<0.05)$ while the levels of GSH and SOD were significantly diminished $(\mathrm{p}<0.05)$ in the EAC group when 
versus to the control group. Treatment of EAC mice with CPA resulted in significant rises $(p<0.05)$ in the hepatic levels of MDA, as well as significant reductions in hepatic activities of GSH and SOD as versus to the control groups. Meanwhile, treatment of EAC mice with BM-MSCs or CPA+BM-MSCs resulted in significant, dosedependent reductions in MDA levels and significant increment in the activities of GSH and SOD verses to both of EAC and EAC+CPA groups.

Table 2: Effects of treatment with CPA or/and BM-MSCs on liver enzymes in liver tissue of EAC mice Effects of BM-MSCs on EAC mice treated with cyclophosphamide (CPA)

\begin{tabular}{|c|c|c|c|}
\hline Groups $\quad$ Parameters & $\begin{array}{l}\text { ALT } \\
(\mathrm{U} / \mathrm{l})\end{array}$ & $\begin{array}{l}\text { AST } \\
(\mathrm{U} / \mathrm{l})\end{array}$ & $\begin{array}{l}\text { GGT } \\
(U / g)\end{array}$ \\
\hline Con & $66.17 \pm 7.28$ & $235.62 \pm 12.35$ & $25.83 \pm 4.05$ \\
\hline \multirow[t]{2}{*}{ EAC } & $97.73 \pm 8.35^{\mathrm{a}}$ & $283.25 \pm 11.82^{\mathrm{a}}$ & $132.62 \pm 12.82^{\mathrm{a}}$ \\
\hline & a $(47.69 \%)$ & a $(20.21 \%)$ & a (413.43\%) \\
\hline \multirow[t]{2}{*}{$\mathrm{EAC}+\mathrm{CPA}$} & $120.62 \pm 9.13^{b}$ & $348.25 \pm 15.31^{b}$ & $118.23 \pm 4.21^{\mathrm{b}}$ \\
\hline & $\mathrm{b}(23.42 \%)$ & b $(22.94 \%)$ & b $(-10.85 \%)$ \\
\hline \multirow[t]{2}{*}{ EAC+BM-MSCs } & $82.08 \pm 6.71^{b}$ & $268.30 \pm 12.62^{b}$ & $62.82 \pm 6.23^{\mathrm{b}}$ \\
\hline & b (-16.01\%) & b $(-5.27 \%)$ & b (-52.63\%) \\
\hline \multirow[t]{3}{*}{$\mathrm{EAC}+\mathrm{CPA}+\mathrm{BM}-\mathrm{MSCs}$} & $72.56 \pm 8.23^{\mathrm{bc}}$ & $252.82 \pm 13.12^{b c}$ & $38.71 \pm 4.32^{\mathrm{bc}}$ \\
\hline & b (-25.75\%) & b (-10.74\%) & b (-70.81\%) \\
\hline & c $(-39.84 \%)$ & c $(-27.40 \%)$ & $c(-67.25 \%)$ \\
\hline
\end{tabular}

a: Significant change at $\mathrm{P}<0.05$ when it compared to the normal control group (Con); b: Significant change at $\mathrm{P}<0.05$ contra EAC group. c: Significant change at $\mathrm{P}<0.05$ contra $\mathrm{EAC}+\mathrm{CPA}$ group.

Table 3: Effects of treatment with CPA or/and BM-MSCs on oxidative stress markers in liver tissue of EAC mice

\begin{tabular}{|c|c|c|c|}
\hline Groups & $\begin{array}{l}\text { MDA } \\
\text { (nmol/gm) }\end{array}$ & $\begin{array}{l}\text { GSH } \\
\text { (mg/g tissue) }\end{array}$ & $\begin{array}{l}\text { SOD } \\
(\mu g / g m)\end{array}$ \\
\hline Con & $86.28 \pm 9.12$ & $56.43 \pm 6.12$ & $338.81 \pm 10.12$ \\
\hline EAC & $\begin{array}{l}165.14 \pm 10.52^{\mathrm{a}} \\
\mathrm{a}(91.40 \%)\end{array}$ & $\begin{array}{l}26.32 \pm 4.41^{a} \\
a(-53.35 \%)\end{array}$ & $\begin{array}{l}226.82 \pm 11.21^{\mathrm{a}} \\
\text { a }(-33.05 \%)\end{array}$ \\
\hline $\mathrm{EAC}+\mathrm{CPA}$ & $\begin{array}{l}282.76 \pm 17.32^{a} \\
\text { b }(71.22 \%)\end{array}$ & $\begin{array}{l}16.11 \pm 2.12^{b} \\
b(-38.79 \%)\end{array}$ & $\begin{array}{l}118.32 \pm 9.24 b \\
b(-47.85 \%)\end{array}$ \\
\hline $\mathrm{EAC}+\mathrm{BM}-\mathrm{MSCs}$ & $\begin{array}{l}132.27 \pm 11.35^{b} \\
b(-19.90 \%)\end{array}$ & $\begin{array}{l}33.02 \pm 5.94 b \\
b(25.45 \%)\end{array}$ & $\begin{array}{l}272.11 \pm 12.61^{b} \\
b(19.96 \%)\end{array}$ \\
\hline $\mathrm{EAC}+\mathrm{CPA}+\mathrm{BM}-\mathrm{MSCs}$ & $\begin{array}{l}99.82 \pm 10.81^{b c} \\
b(-39.55 \%) \\
c(-64.69 \%)\end{array}$ & $\begin{array}{l}45.51 \pm 5.11^{b c} \\
\mathrm{~b}(72.91 \%) \\
\mathrm{c}(182.49 \%)\end{array}$ & $\begin{array}{l}315.25 \pm 13.03^{\mathrm{bc}} \\
\mathrm{b}(38.98 \%) \\
\mathrm{c}(166.44 \%)\end{array}$ \\
\hline
\end{tabular}

a: Significant change at $\mathrm{P}<0.05$ when it compared to the normal control group (Con); b: Significant change at P<0.05 contra EAC group. c: Significant change at $\mathrm{P}<0.05$ contra $\mathrm{EAC}+\mathrm{CPA}$ group.

Effects of treatment with CPA or/and BM-MSCs on Inflammatory and Anti-inflammatory mediators in EAC mice

The result of this study showed that hepatic IL-6 level displayed significant elevation $(\mathrm{P}<0.05)$ in the EAC treated group correlated with Maximum amelioration in the level of IL-10 as compared with the control groups proving increased inflammatory response (table 4). However, CPA treatment induced upregulation in the level of IL-6 and downregulation in the level of IL-10 as compared with the EAC group. In contrast, treatment of EAC group with BM-MSCs or CPA+BM-MSCs significantly restored the levels of IL- 6 and IL-10 near to normal levels compared to both EAC and EAC+CPA groups (table 4).

Table 4: Effects of treatment with CPA or/and BM-MSCs on inflammatory and anti-inflammatory markers in liver tissue of EAC mice

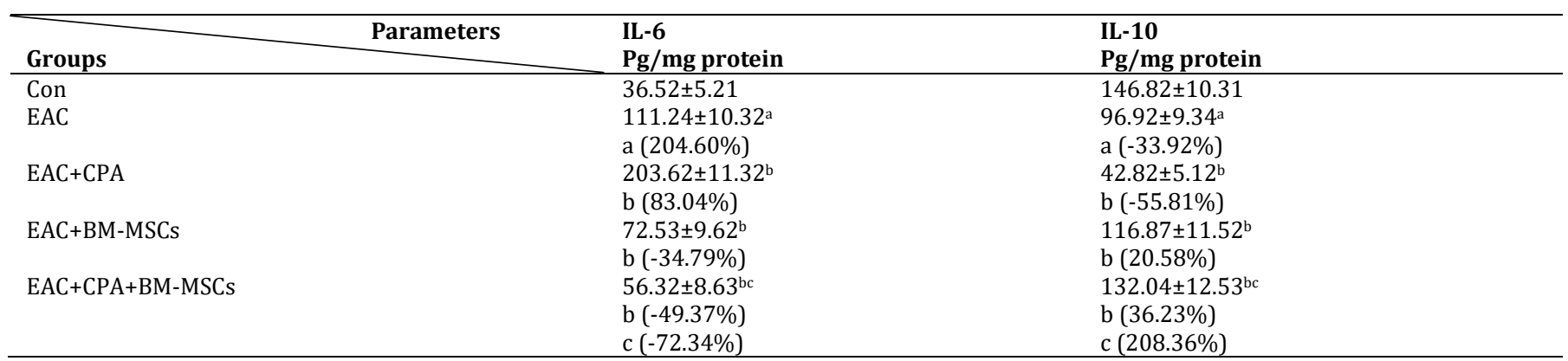

a: Significant change at $\mathrm{P}<0.05$ when it compared to the normal control group (Con); b: Significant change at $\mathrm{P}<0.05$ contra EAC group. c: Significant change at $\mathrm{P}<0.05$ contra EAC+CPA group.

Effects of treatment with CPA or/and BM-MSCs on apoptotic and antiapoptotic markers in EAC mice

It is clear from table 5 that the inoculation of EAC into mice induced liver injury caused a marked increase $(\mathrm{P}<0.05)$ in the level of Caspase-3 indicating acceleration of apoptosis reflected by significant depleting in the hepatic levels of $\mathrm{Bcl}_{2}$ compared to the normal control group.

CPA administration increased significantly $(\mathrm{P}<0.05)$ the level of IL- 6 and decreases significantly $(\mathrm{P}<0.05)$ the levels of Caspase- 3 as compared to the EAC group. 
However, decreased levels of Caspase-3 and increased levels $(\mathrm{P}<0.05)$ of $\mathrm{Bcl}_{2}$ were recorded following the treatment of
EAC group with BM-MSCs or CPA and BM-MSCs together compared to both of EAC and EAC+CPA groups (table 5).

Table 5: Effects of treatment with CPA or/and BM-MSCs on apoptotic and anti-apoptotic markers in liver tissue of EAC mice

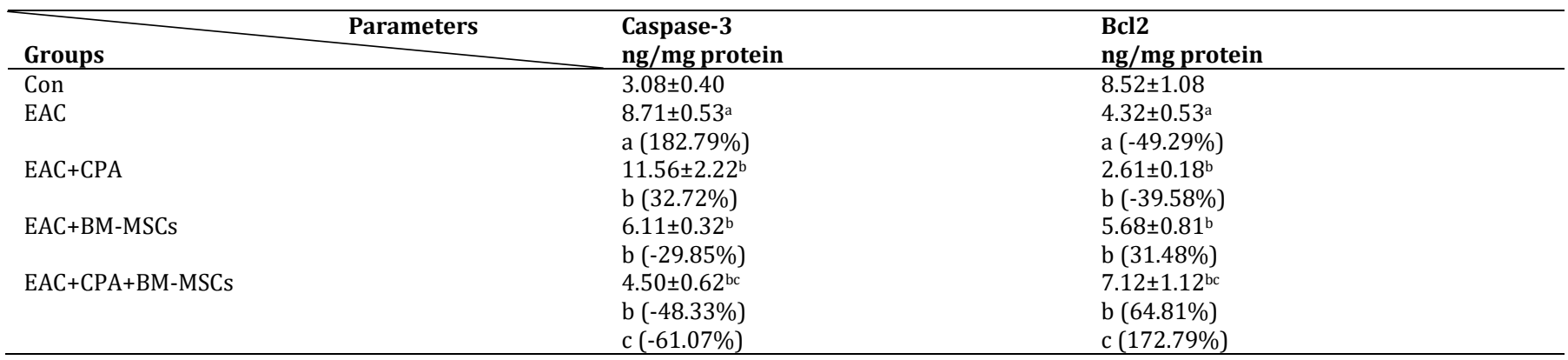

a: Significant change at $\mathrm{P}<0.05$ when it compared to the normal control group (Con); b: Significant change at $\mathrm{P}<0.05$ contra EAC group. c: Significant change at $\mathrm{P}<0.05$ contra $\mathrm{EAC}+\mathrm{CPA}$ group.

\section{Histopathological investigation}

Histopathological studies showed that inoculation of EAC into mice induced massive liver damage including severe congestion of erythrocytes and leucocytes, leucocytic infiltration, and vacuolar degeneration when compared to the control rat liver (fig. 6B). Also, dilatation in the portal vein and Thickening in the bile ductule wall was observed due to the accumulation of fibers around the wall lining (fig. 6C). On the other side, treatment with CPA revealed some changes in the liver tissue demonstrated by dilatation in the portal vein and destruction in its wall beside leucocytic infiltration surrounded by necrotic lesions was noted. Moreover, different types of leucocytes appeared scattered in the tissue besides giant phagocytic Kupffer cells in addition to large foci of necrosis (fig. 6D and E). The pathological changes induced by EAC were improved greatly in the liver of rats treated with BM-MSCs (fig. 6F). However, after the treatment with CPA+BM-MSCs showed the hepatic lobules appeared more like normal and hepatic cells are arranged in a series of branching plates (fig. 6G).

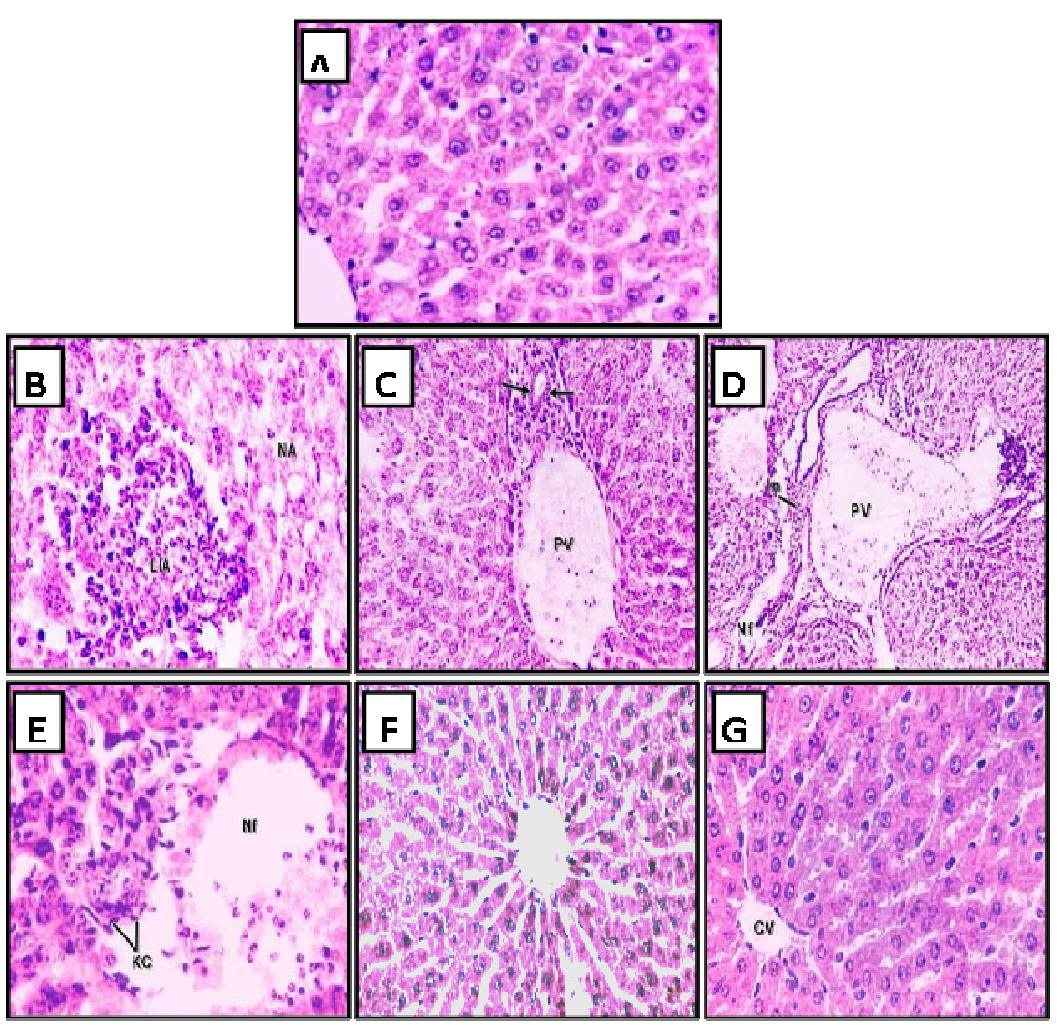

Fig. 6: (A) photomicrographs of $\mathrm{H}$ and $\mathrm{E}$ stained liver sections of control mice demonstrated the normal hepatic architecture. (X200). (B) hepatic Section of EAC mice displayed distortion in hepatic architecture manifested by leucocytic infiltration area (LIA), necrotic areas

(NA) and degeneration of the liver cells that looked empty of nucleus (X400). (C)Liver Section of EAC mice showing swollen in lining epithelia of the bile ductule (arrows), congestion of erythrocytes and leucocytes in portal vein (PV) and leucocytic infiltration (X200). (D) Liver Section of EAC mice treated with CP showing dilatation in portal vein (PV), Leucocytic infiltration (In) and necrotic foci (Nf) (X100). (E) Liver Section of EAC mice treated with CP displayed large necrotic foci (Nf) with scattered inflammatory cells and giant Kupffer cells (KC) (X400). (F) Hepatic section of EAC mice treated with BM-MSCs showed almost normal hepato-cellular architecture (X100). (G) Liver Section of EAC mice treated with CP+BM-MSCs displayed normal healthy hepatocytes around normal central vein (CV) (X200). 


\section{DISCUSSION}

The current study showed that CPA, BM-MSCs or CPA and BM-MSCs administration significantly reduced the solid tumor burden in mice and enhanced the survival time of EAC bearing mice, especially in the co-administration of CPA and BM-MSCs which caused an increase in the life span of animals and the tumor inhibition ratio [28]. The present findings revealed that both CPA and BM-MSCs exerted antitumor activity through interference with cell metabolism and inhibiting tumor proliferation. CPA undergoes metabolic activation by hepatic enzymes and forms 4-hydroxy cyclophosphamide, which converts into two cytotoxic metabolites acrolein and phosphoramide mustard. These cytotoxic metabolites on enzymatic activation form covalent bonds with DNA and proteins, causing DNA damage, This DNA damage could render tumor cells more apt to undergo apoptosis causing cell death, hence reducing the proliferation, migration and invasive properties of the cell line [29]. BM-MSCs repressed tumor proliferation and metastasis via altitude T-cell responses likely through MSC-secreted T-cell chemokines. All through increasing T-cell responses, the excessive levels of T-cell cytokines subsequently turn on the negative feedback mechanisms in MSCs to suppress excessive T-cell reactivity. A key Tcell cytokine in this regulation is IFN $\gamma$, which acts independently or with inflammatory mediators including tumor necrosis factor- $\alpha$ (TNF- $\alpha$ ), IL-1 and interleukin 17 to functionally transform MSCs from a resting state to a highly immunosuppressive stage [30]. Such immunosuppression is attained via cytokine-induced manifestation of the immunosuppressive molecules inducible nitric oxide synthase (iNOS) in human and mouse MSCs [31].

The results of the present study also demonstrated that the coadministration of CPA and BM-MSCs resulted in a significant decrease in MTW and increases the mean survival time of tumorbearing mice. This decrease in MTW may attribute to the action of CPA in the development of free radicals-mediated oxidative stress in tumor cells. This ROS production is believed to be the main effect in sensitizing tumor cells towards apoptosis [32]. Compatible with our results, a preceding study found that exosomes derived from microRNA-143-overexpressing mesenchymal stem cells inhibiting proliferation, invasion, and migration while enhancing apoptosis of hepatoma cells via regulating the NF- $\kappa B$ pathway [33].

In the present study, the elevations recorded in serum ALT, AST and GGT activities in EAC and CPA-treated groups may be due to drastic conditions induced by the toxic activity of tumor cells and CPA accumulations in the liver and in turn this might cause cellular destruction or increase hepatic cells permeability [34]. In contrast, the EAC group treated with BM-MSCs recorded a significant decline in serum ALT, AST and GGT activities as versus to the EAC group. Moreover, the double treatment of EAC with both of CPA and BM-MSCs resulted in a significant improvement in serum ALT, AST and GGT as compared to both EAC group and $\mathrm{EAC}+\mathrm{CPA}$ group, showing the protective effects of BM-MSCs against liver injury and hepatic fibrosis. Several studies demonstrated that transplantation of stem cells has been demonstrated to exert therapeutic effects in cases of liver fibrosis, as well as after repeated partial hepatectomy, and significantly lowering levels of AST, ALT, and GGT [35]. The acquired results exhibited a significant increase of hepatic MDA level in tumor-bearing mice associated with a significant decline in GSH and SOD contents as versus to that of the normal control group. Such finding is in agreement with Fahim et al. [19] who stated that the increase in MDA and decrease of GSH and SOD due to the oxidative stress on the host in response to the continual production of free radicals by the expanding tumor load. The present work demonstrated a significant increase in hepatic MDA level correlated with a significant decrease in SOD activity as well as GSH level in the EAC+CPA group as compared to EAC group. Decreasing of the antioxidant levels may be due to malfunction of the antioxidant defense mechanisms that probably result from the consumption of antioxidants by chemotherapy stimulated-oxidative stress and/or renal loss of low molecular weight, water soluble antioxidants. Maruthanila et al. [36] stated that the prooxidant properties of chemotherapy may be implicated in its capability to induce tumor cell apoptosis, which is achieved partly through direct oxidative damage of DNA, RNA, and/or protein in the cells. Current study revealed an improvement by BM-MSCs treatment in the hepatic MDA, GSH and SOD levels in the both of EAC group and EAC+CPA+BM-MSCs versus to EAC group and $\mathrm{EAC}+\mathrm{CPA}$ group. BM-MSCs rise GSH via increased efflux of cysteine with enhanced GSH synthesis which facilitated by secretion of soluble growth factors by BM-MSCs or by their interaction with host cells or both [37]. Likewise, our results show that BM-MSCs infusion is associated with expansion of SOD activity which reflects the efficiency of BM-MSCs in combating the induced oxidative stress [38]. This study is built on the fact that stem cells can be chemotactically attracted to the location of injury [39].

In the contemporary study, implantation of EAC resulted in a significant increment in IL- 6 associated with a significant reductions in IL-10 versus to the control group which was in a similar line with other studies that stated that proinflammatory cytokines such as IL6 have an important role in cancer development [40]. IL-6 is possibly the best characterized protumorigenic cytokine that was initially supposed to be complicated in cancer due to their capability to activate the oncogenic transcription factors such as activator protein 1 (AP-1), signal transducers and nuclear factor kappa B (NF$\mathrm{kB}$ ) and activators of transcription 3 (STAT3) in epithelial cells [41].

The kindness impacts of IL-10 in cancer include inhibition of angiogenesis either directly on tumor cells or indirectly boosts antitumor immunity by influencing infiltrating immune cells [42] and modulation of apoptosis [43]. IL-10 may wield pro-tumorigenic effect via repression of adaptive immune replies that caused tumor escape from immune surveillance [44]. These opposite effects of IL-10 might depend on relations with either cytokines or factors found in the tumor microenvironment. In this perspective, IL-10 has been stated to repress NO production and control the inducible NO synthase (iNOS) activation [45]. The iNOS-derived NO has been identified as one of the extremely versatile players in the immune system and pathogenesis of numerous diseases as well as cancer [46].

Administration of CPA resulted in hepatotoxicity manifested by a significant increase in IL- 6 associated with a significant decrease in the IL-10 as compared to the EAC group. These results reveal the existence of liver inflammation in mice treated with CPA. Inflammation is the most important cause of liver injury, as various pieces of substantiation indicate that high levels of inflammatory cytokines can damage the liver [47]. Pro-inflammatory cytokines, for example, interleukin (IL)-1b and IL-6 can injure the hepatocytes via the NF-kB pathway. Simultaneously, the NF-kB pathway controls the synthesis and expression of numerous inflammatory factors and then produces apoptosis of the hepatocytes. Studies have demonstrated that CPA can promote the IL-1b, IL- 6 and TNF- $\alpha$ levels [48]. Furthermore, apart from the influence of CPA and its metabolites on the IL- 6 production the diminishing of IL-10 production by macrophages [49].

On the other hand, the treatment of the EAC group and EAC+CPA+BM-MSCs with BM-MSCs recorded a significant reduction in the IL-6 level associated with significant increment in IL-10 level as compared to the EAC group and EAC+CPA group. MSCs are recognized for their powerful anti-inflammatory functions, as they habitually transplanted into inflammatory environments where they capable to survive and regulate host immune responses [50]. The gained findings of the existing study declare that the protective influence of BM-MSCs is multifactorial as well as oxidative stress, inflammatory response, and tissue repair. It has been stated that MSCs are qualified for delivering an assortment of cytokines and hematopoietic soluble growth factors [51].

In this study, EAC inoculation produced a significant increment in hepatic caspase 3 and significant reduction in the hepatic Bcl2 level versus to normal control group. It is known that damaged cells could tend to turn into cancer if they did not choose apoptosis [52]. Caspase 3 activity likewise raised in human hepatocarcinoma in vitro and in vivo studies by S. marianum treatment [53]. EAC apoptotic cellular death stimulated by activating caspase 3 utilizing Synadenium umbellatum Pax [54]. In view of our findings, Badr El-Din et al. [55] documented that significant variations in the expression of apoptotic regulators (activation of caspase-3, upregulation in the expression of proapoptotic proteins Bax and p53, downregulation in the expression of the antiapoptotic protein Bcl-2, and decreased Bcl2: Bax ratio) in mice that were inoculated with Ehrlich ascites carcinoma. The treatment of EAC group with CPA was also up-regulating the hepatic level of caspase- 3 and 
down-regulating the hepatic level of $\mathrm{Bcl}-2$ as compared to the EAC group. Related studies Shokrzadeh et al. [8] reported that the CPA-induced liver damage was associated with the ability to regulate hepatocyte apoptosis, including DNA insertion, p53 protein activation, and ROS production. Initiation of p53 further begins a cascade of enzymatic reactions that push along to stimulate downstream members of the family (such as caspase- 3 and caspase-9) even though a positive feedback process. Stimulated caspase- 3 specifically cleaves its substrate and fragments DNA to produce apoptosis [56]. Caspase-3 and caspase- 9 can be initiated, and apoptosis can be caused by regulating the expression of mitochondrial apoptosis-related proteins (eg. Bcl-2 or Bax) or causing apoptosis-related complexes [57].

In contrast, the treatment of the EAC group and EAC+CPA+BM-MSCs with BM-MSCs recorded a significant reduction in the caspase-3 level associated with significant increment in Bcl-2 level as compared to the EAC group and EAC+CPA group. BM-MSCs considerably diminished caspase- 3 activity, this finding verified their role in mitigating inflammatory reply and apoptosis to healing damaged cells and BM-MSCs are of a certain therapeutic effect for hepatic dysfunction [38]. It has been described that apart from cytokine/chemokine secretion, MSCs also exhibit a powerful capacity for mitochondrial transfer and microvesicle (exosomes) emission in response to injury with consequent advancement of tissue regeneration [58]. Exosomes have a most important role in the paracrine impact of transplanted cells where they be able to carry a complex content that involves nucleic acids proteins long noncoding RNAs and microRNA [59]. Feng et al. [60] displayed that MSCs-derived exosomes include miR-22, which silence methyl CpG binding protein 2 and lessened apoptosis in cardiac tissue. Additionally, numerous studies should be performed to establish the vital role of cytokines, growth factor and MSCs released vesicles (exosomes) in purpose repair of MSCs in addition to establishing their role in hepatic microenvironment.

The present study showed that Ehrlich solid tumor-bearing mice showed harmful effects on liver tissue displayed by degeneration of the liver cells and dilatation in the portal vein beside marked congestion of erythrocytes and leucocytes surrounded by Aggregations of inflammatory cells. The infiltrations of Ehrlich tumor cells are thought to be the proliferation and migration of tumor cells into the internal organs and the presence of Aggregations of inflammatory cells might occur to degeneration of the mitochondria or disorganization of the cytoplasm [61]. Meanwhile, CPA showed major impairments dilatation in the portal vein and marked necrotic area surrounded by inflammatory cells and giant Kupffer cells in the tissue. This observation coincided with the results of Rahmouni et al. [62] who found that hepatic centrilobular necrosis, degeneration, and infiltration in the liver of CPA-intoxicated animals is associated with A higher level of liver marker enzymes in the bloodstream. On the other side, co-treatment of BM-MSCs with CPA compensated the conspicuous hepatic alterations induced by EAC and CPA and restored the liver tissue to normal hepato-cellular architecture indicating to anti-inflammatory, antiproliferative and anti-fibrotic effect of BM-MSCs [63].

\section{CONCLUSION}

In conclusion, these findings demonstrated that BM-MSCs might be significantly reducing CPA-induced hepatotoxicity in EAC mice. This protection of BM-MSCs was demonstrated by the induction of the antioxidant enzyme systems to increase the disposal of overproduction reactive free oxygen radicals by blocking the lipid peroxidation in liver tissue after CPA treatment. Moreover, this study revealed that BM-MSCs significantly ameliorate the inflammation and apoptosis in liver tissue characterizing hepatotoxicity in EAC mice treated with CPA due to its antiinflammatory effect as well as its antiapoptotic effect. In addition, BM-MSCs enhance the antitumor properties of CPA by increase the inhibitory effect of CPA on tumor growth.

\section{FUNDING}

This research did not receive any specific grant from funding agencies in the public, commercial, or not-for-profit sectors.

\section{AUTHORS CONTRIBUTIONS}

Asmaa M Zaazaa and Bosy A Abd El-Motelp created, designed the study, analyzed the data, wrote and revised the manuscript.

\section{CONFLICT OF INTERESTS}

The authors declare that they have no conflicts of interest that influenced this work.

\section{REFERENCES}

1. Abbas S, Malla S. Cytotoxicity and expression studies of angiogenesis-promoting genes in cancer cell lines under the treatment of cancer candidate drugs. Asian J Pharm Clin Res 2019;12:130-4.

2. Barakat W, Elshazly SM, Mahmoud AA. Spirulina platensis lacks antitumor effect against Solid Ehrlich Carcinoma in female mice. Adv Pharmacol 2015. Doi:10.1155/2015/132873

3. Sinanoglu O, Yener AN, Ekici S, Midi A, Aksungar FB. The protective effects of spirulina in cyclophosphamide induced nephrotoxicity and urotoxicity in rats. Urology 2012;80:1392-6.

4. Jiang W, Liu J, Li P, Lu Q, Pei X, Sun Y, et al. Magnesium iso glycyrrhizinate shows hepatoprotective effects in a cyclophosphamide induced model of hepatic injury. Oncotarget 2017;8:33252-64.

5. Kim J, Chen $\mathrm{CH}$, Yang J, Mochly Rosen D. Aldehyde dehydrogenase $2 * 2$ knock-in mice show increased reactive oxygen species production in response to cisplatin treatment. J Biomed Sci 2017;24:33.

6. Mahmoud AM, Germoush MO, Alotaibi MF, Hussein OE. Possible involvement of Nrf2 and PPAR $\gamma$ up-regulation in the protective effect of umbelliferone against cyclophosphamide-induced hepatotoxicity. Biomed Pharmacother 2017;86:297-306.

7. Subramaniam SR, Cader RA, Mohd R, Yen KW, Ghafor HA. Lowdose cyclophosphamide-induced acute hepatotoxicity. Am J Case Rep 2013;14:345.

8. Shokrzadeh M, Ahmadi A, Naghshvar F, Chabra A, Jafarinejhad M. Prophylactic efficacy of melatonin on cyclophosphamideinduced liver toxicity in mice. Bio Med Res Int 2014. https://doi.org/10.1155/2014/470425

9. Jalali AS, Hasanzadeh S, Malekinejad H. Achillea millefolium inflorescence aqueous extract ameliorates cyclophosphamideinduced toxicity in rat testis: stereological evidences. Chin J Nat Med 2012;1:247-54.

10. Abd El-Fattah AI, Zaghloul MS, Eltablawy NA, Rashed LA. a-Lipoic acid and amlodipine/perindopril combination potentiate the therapeutic effect of mesenchymal stem cells on isoproterenol induced cardiac injury in rats. Biochimie 2019;156:59-68.

11. Caplan AL, Dennis JE. Mesenchymal stem cells as trophic mediators. J Cell Biochem 2006;98:1076-84.

12. Vincent PC, Nicholls A. Comparison of the growth of the Ehrlich ascites tumor in male and female mice. Cancer Res 1967;27:1058-65.

13. Strober W. Trypan blue exclusion test of cell viability. Curr Protoc Immunol 2001;21:A.3B.1-2.

14. Abdel Aziz M, Atta H, Mahfouz S, Fouad H, Roshdy N, Ahmed H, et al. Therapeutic potential of bone marrow derived mesenchymal stem cells on experimental liver fibrosis. Clin Biochem 2007;40:893-9.

15. Jaiswal N, Haynesworth S, Caplan A, Bruder S. Osteogenic differentiation of purified, culture-expanded human mesenchymal stem cells in vitro. J Cell Biochem 1997;64:295-312.

16. Perry MJ. The chemotherapy source book. Wolters Kluwer Health/lippincott Williams and Wilkins. Philadelphia; 2008.

17. Agrawal SS, Saraswati S, Mathur R, Pandey M. Cytotoxic and antitumor effects of brucine on Ehrlich ascites tumour and human cancer cell line. Life Sci 2011;89:147-58.

18. El Gammal ZH, Rashed LA, Abdel Aziz MT, Ahmed H, Elwahy M, Youakim MF, et al. Comparative study between the attenuation of cardiac fibrosis by mesenchymal stem cells versus colchicine. Acta Med Int 2016;3:137.

19. Fahim FA, Esmat AY, Mady EA, Ibrahim EK. Antitumor activities of Iodo acetate and Di methyl sulphoxide against solid Ehrlich carcinoma growth in mice. Biol Res 2003;36:253-62. 
20. Lowry OH, Rosebrough NJ, Farr AL, Randall RJ. Protein measurement with the Folin phenol reagent. J Biol Chem 1951;193:265-75.

21. Young DS. Effect of drugs on clinical laboratory tests. 3rd ed. Washington DC: AACC Press; 1990. p. 6-12.

22. Satoh K. Serum lipid peroxide in cerebrovascular disorders determined by a new colorimetric method. Clin Chim Acta 1978;90:37-43.

23. Beutlr E, Duron O, Kelly BM. Improved method for the determination of blood glutathione. J Lab Clin Med 1963;61:882-8.

24. Nishikimi M, Appaji N, Yagi K. The occurrence of superoxide anion in the reaction of reduced phenazine methosulfate and molecular oxygen. Biochem Biophys Res Commun 1972;46:849-54.

25. Barbareschi $M$, Veronese $S$, Leek $R$, Fox $S$, Bonzanini $M$, Girlando S. Bcl-2 and P53 expression in node-negative breast carcinoma-a study with long-term follow up. Hum Pathol 1996;27:1149-55.

26. Banchroft JD, Stevens A, Turner DR. Theory and practice of histological techniques. 4th ed. New York, London, San Francisco, Tokyo: Churchil Livingstone; 1996.

27. Abramoff MD, Magalhaes PJ, Ram SJ. Image processing with image. J Biophotonics Int 2004;11:36-42.

28. Agrawal SS, Sharma P. Anticancer activity of cyclophosphamide nanoparticles against ehrlich ascites carcinoma cells bearing swiss albino mice. Inter J Pharm Pharm Res 2017;9:244-65.

29. Mahipal P, Pawar RS. Nephroprotective effect of Murraya koenigii on cyclophosphamide induced nephrotoxicity in rats. Asian Pac J Trop Med 2017;10:869-73.

30. Han $\mathrm{X}$, Yang $\mathrm{Q}$, Lin $\mathrm{L}, \mathrm{Xu} \mathrm{C}$, Zheng $\mathrm{C}$, Chen $\mathrm{X}$, et al. Interleukin-17 enhances immunosuppression by mesenchymal stem cells. Cell Death Differentiation 2014;21:1758-68.

31. Su J, Chen X, Huang Y, Li W, Li J, Cao K, et al. Phylogenetic distinction of iNOS and IDO function in mesenchymal stem cellmediated immunosuppression in mammalian species. Cell Death Differentiation 2014;21:388-96.

32. Chakraborty AP, Singha Roya PA, Basua A, Bhattacharyaa S. Sensitization of cancer cells to cyclophosphamide therapy by an organo-selenium compound through ROS-mediated apoptosis. Biomed Pharmacother 2016;841:992-9.

33. Zheng $\mathrm{Y}$, Yang $\mathrm{F}, \mathrm{Fu} \mathrm{L}$, Liu $\mathrm{K}$. The mechanism of miR-143 inducing apoptosis of liver carcinoma cells through regulation of the NF-kappaB pathway. Oncol Lett 2018;15:9567-71.

34. Donia TIK, Gerges MN, Mohamed TM. Amelioration effect of Egyptian sweet orange hesperidin on Ehrlich ascites carcinoma (EAC) bearing mice. Chem Biol Interact 2018;285:76-84.

35. Apostolou G, Papanikolaou IG, Katselis C, Feretis T, Kletsas D, Konstadoulakis MM, et al. Undifferentiated adipose tissue stem cell transplantation promotes hepatic regeneration, ameliorates histopathologic damage of the liver, and upregulates the expression of liver regeneration-and liverspecific genes in a rat model of partial hepatectomy. Stem Cells Int 2018;1-18. Doi:10.1155/2018/1393607

36. Maruthanila VL, Poornima J, Mirunalini S. Attenuation of carcinogenesis and the mechanism underlying by the influence of indole-3-carbinol and its metabolite 3, 3'-diindolylmethane: a therapeutic marvel. Adv Pharmacol Sci 2014. Doi:10.1155/2014/832161

37. Iyer SS, Torres Gonzalej E, Neujahr DC, Kwon M, Brigham KL, Jones DP, et al. Effect of bone marrow-derived mesenchymal stem cells on endotoxin-induced oxidation of plasma cysteine and glutathione in mice. Stem Cell Int 2010. https://doi.org/10.4061/2010/868076

38. Zhang M, Li DS, Chang L, Zhang Y, Liu R, Sun F, et al. Bone marrow mesenchymal stem cell transplantation retards the natural senescence of rat hearts. Stem Cell Transl Med 2015;4:494.

39. Willing AE, Lixian J, Milliken M, Poulos S, Zigova T, Song S, et al. Intravenous versus intrastriatal cord blood administration in a rodent model of stroke. J Neurosci Res 2003;73:296.

40. Waldner MJ, Foersch S, Neurath MF. Interleukin-6-a key regulator of colorectal cancer development. Int J Biol Sci 2012;8:1248-53.

41. Grivennikov SI, Karin M. Inflammatory cytokines in cancer: tumour necrosis factor and interleukin 6 take the stage. Ann Rheum Dis 2011;70:i104-8.
42. Blankenstein $\mathrm{T}$. The role oftumor stroma in the interaction between tumor and immune. Curr Opin Immunol 2005;17:180-6

43. Mocellin S, Marincola FM, Young HA. Interleukin-10 and the immune response against cancer: a counterpoint. J Leukoc Biol 2005;78:1043-51.

44. Moore KW, de Waal Malefyt R, Coffman RL, O'Garra A. Interleukin-10 and the interleukin-10 receptor. Annu Rev Immunol 2001;19:683-765.

45. Gazzinelli RT, Oswald IP, James SL, Sher A. IL-10 inhibits parasite killing and nitrogen oxide production by IFNgamma-activated macrophages. J Immunol 1992;148: 1792-6.

46. Lechner M, Lirk P, Rieder J. Inducible nitric oxide synthase (iNOS) in tumor biology: the two sides of the same coin. Semin Cancer Biol 2005;15:277-89.

47. Trombetta D, Cimino F, Cristani M, Mandalari G, Saija A, Ginestra $G$, et al. In vitro protective effects of two extracts from bergamot peels on human endothelial cells exposed to tumor necrosis factor-a (TNF-a). J Agric Food Chem 2010;58:8430-6.

48. Kumar S, Dhankhar N, Kar V, Shrivastava M, Shrivastava S. Myocardial injury provoked by cyclophosphamide, protective aspect of hesperidin in rats. Int J Res Pharm Biomed Sci 2011;2:1288-96.

49. Bryniarski K. The influence of cyclophosphamide on immune function of murine macrophages. Pharmacology 2012;143-60. Doi:10.5772/32891

50. Carrero R, Cerrada I, Lledo E, Dopazo J, Garcia Garcia F, Rubio $M$, et al. IL1 $\beta$ induces mesenchymal stem cells migration and leucocyte chemotaxis through NF- $\kappa \beta$. Stem Cell Rev Rep 2012;8:905-16.

51. Liang X, Ding Y, Zhang Y, Tse HF, Lian Q. Paracrine mechanisms of mesenchymal stem cell-based therapy: current status and perspectives. Cell Transplant 2014;23:1045.

52. You P, Xing F, Huo J, Wang B, Di J, Zeng S, et al. In vitro and in vivo evaluation of anisomycin against Ehrlich ascites carcinoma. Oncol Rep 2013;29:2227-36.

53. Zhang S, Yang Y, Liang Z, Duan W, Yang J, Yan J, et al. Silybinmediated inhibition of Notch signaling exerts antitumor activity in human hepatocellular carcinoma cells. PLOS ONE 2013;8:e83699.

54. Da Mota MF, Benfica PL, Batista AC, Martins FS, de Paula JR, Valadares MC. Investigation of Ehrlich ascites tumor cell death mechanisms induced by Synadenium umbellatum. Pax J Ethnopharmacol 2012;139:319-29.

55. Badr El-Din NK, Ali DA, Abou-El-Magd RF. Grape seeds and skin induce tumor growth inhibition via G1-phase arrest and apoptosis in mice inoculated with Ehrlich ascites carcinoma. Nutrition 2019;58:100-9.

56. Brentnall M, Rodriguez Menocal L, De Guevara RL, Cepero E, Boise LH. Caspase-9, caspase- 3 and caspase-7 have distinct roles during intrinsic apoptosis. BMC Cell Biol 2013;14:32.

57. Xu LX, Yan LJ, Huang SP. Ganoderic acid A against cyclophosphamide induced hepatic toxicity in mice. J Biochem Mol Toxicol 2019;33:e22271.

58. Chimenti I, Smith RR, Li TS, Gerstenblith G, Messina E, Giacomello A, et al. An relative roles of direct regeneration versus paracrine effects of human cardiosphere-derived cells transplanted into infarcted mice. Circ Res 2010;106:971.

59. Hirsch E, Hilfiker Kleiner D, Balligand JL. Interaction of the heart and its close and distant neighbours: report of the meeting of the esc working groups myocardial function and cellular biology. Cardiovasc Res 2013;99:595.

60. Feng Y, Huang W, Wani M. Ischemic preconditioning potentiates the protective effect of stem cells through secretion of exosomes by targeting Mecp2 via miR-22. PloS One 2014;9:e88685.

61. Ali DA, Badr El-Din NK, Abou-El-magd RF. Antioxidant and hepatoprotective activities of grape seeds and skin against Ehrlich solid tumor induced oxidative stress in mice. Egyptian J Basic Appl Sci 2015;2:98-109. 
62. Rahmouni F, Hamdaoui L, Badraoui R, Rebai T. Protective effects of Teucrium polium aqueous extract and ascorbic acid on hematological and some biochemical parameters against carbon tetrachloride (CCl4) induced toxicity in rats. Biomed Pharmacother 2017;9:43-8.
63. Chen Q, Zhouc R, Zhanga Y, Zhua S, Xiaob C, Gonge J, et al. Bone marrow mesenchymal stromal cells attenuate liver allograft rejection may via upregulation PD-L1 expression through downregulation of miR-17-5p. Transplant Immunol 2018;51:21-9. 